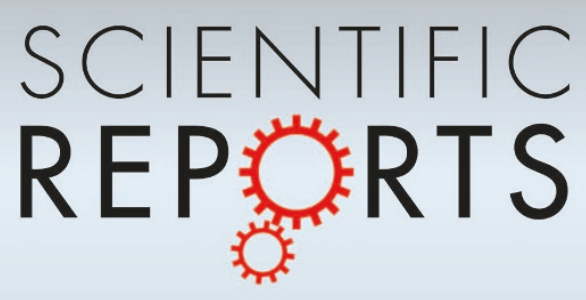

\title{
OPEN 2D Graphene Oxide Nanosheets as an Adhesive Over-Coating Layer for Flexible \\ SUBJECT AREAS: \\ GRAPHENE Transparent Conductive Electrodes
}

MECHANICAL AND STRUCTURAL PROPERTIES AND DEVICES

NANOWIRES

CHEMICAL PHYSICS

Received

11 December 2012

Accepted

11 December 2012

Published

23 January 2013

Correspondence and requests for materials should be addressed to

W.C.K. (woonchun. kim@samsung.com) or H.L. (hyoyoung@skku. edu)

\footnotetext{
* These authors contributed equally to
} this work.

\author{
In Kyu Moon ${ }^{1 *}$, Jae Il Kim²*, Hanleem Lee ${ }^{3 *}$, Kangheon Hur ${ }^{2}$, Woon Chun Kim² \& Hyoyoung Lee ${ }^{1,3}$
}

\begin{abstract}
${ }^{1}$ Center for Smart Molecular Memory, Department of Chemistry, Sungkyunkwan University, Suwon-si 440-746, Republic of Korea, ${ }^{2}$ Corporate R\&D Institute, Samsung Electro-Mechanics, Suwon-si 443-743, Republic of Korea, ${ }^{3}$ Center for Smart Molecular Memory, Department of Energy Science, Sungkyunkwan University, Suwon-si 440-746, Republic of Korea.
\end{abstract}

In recent, highly transparent and flexible, two-dimensional (2D) graphene oxide (GO) nanosheet has been paid attention for various applications. Due to an existence of a large amount of oxygen functional groups, the single 2D GO nanosheet has an insulating, transparent, highly dispersible in the eco-friendly water, and hydrophilic property that has strong adhesion to the hydrophilic surface, which will be the best candidate for the use of an over-coating layer (OCL) and protecting layer for a conductive nanowire based indium-free transparent conductive film (TCF). The ultrathin 2D adhesive GO OCL nanosheet is expected to tightly hold silver nanowires (AgNWs), reduce sheet resistance and produce uniform TCF, providing complete solution that simultaneously solves a high haze, low transparency with a conventional OCL and mechanical instability in cases without a thick OCL. Our novel 2D insulating and hydrophilic GO OCL successfully provided a large-area, flexible, and highly transparent AgNW TCF.

T he preparation of ultrathin 2D GO nanosheet is easy, simple, very cheap, and mass-producible ${ }^{1,2}$. A few GO layers can be easily and cheaply prepared using a solution process, and their thickness can be easily controlled by varying the solution concentrations. The hydrophilicity of 2D GO produce a strong electrostatic adhesion to a hydrophilically pre-treated PET substrate ${ }^{3,4}$, which can tightly hold between the substrate and $2 \mathrm{D}$ nanosheet layer, allowing to be used as an adhesive OCL. As we know, a good OCL has to be an ultra-thin layer so that the nanowire (NW) electrode covered with OCL is flexible and highly transparent, and the OCL should have strong adhesion to a substrate to tightly hold the NW and sharply reduce its sheet resistance, producing high uniform sheet resistance film while providing low haze, high transparency, and low $R_{\mathrm{sh}}$ values with high mechanical stability. In addition, the ultrathin OCL should have an insulating property that allows a direct tunnelling current only through the ultrathin insulating layer. Unlike the conducting hydrophobic reduced graphene oxide (rGO) OCL that did not adhere to the plastic substrate and required an additional OCL that needed chemical or thermal reduction from the $\mathrm{GOs}^{5}$, the insulating GOs do not require patterning of the OCL for device fabrication. Until now, the use of current OCL on transparent, flexible AgNW film causes a sharp increase in the $R_{\mathrm{sh}}$ value from $\sim 8-30$ to $\sim 150-250 \Omega \cdot \mathrm{sq}^{-16,7,8,12,13}$. To overcome simultaneously the problematic issues that arise from the use of a conventional OCL ${ }^{9-13}$, including high haze, low transparency ( $83 \%$ transparency, $\sim 30 \Omega \cdot \mathrm{sq}^{-1}$ as rGO $\mathrm{OCL})^{5}$, high $R_{\mathrm{sh}}$ values and device instability, novel $2 \mathrm{D}$ adhesive OCL should be introduced. Thus, the ultrathin, insulating, highly transparent, flexible, highly dispersible 2D GO nanosheets can be the best candidate as the OCL of NW electrodes such as AgNW and carbon nanotube.

\section{Results}

Fabrication of 2D GO/AgNW/PET film. We propose a new approach to prepare novel AgNW TCF using aqueous GO nanosheets as an OCL on the AgNW films via all-solution and low temperature processing (Fig. 1a). The 2D GO/AgNW/PET film was fabricated using a two-step procedure: bar- or spray-coating of an AgNW solution onto a polyethyleneterephthalete (PET) substrate, followed by spray-coating of the GOs. To improve the adhesion of the hydrophilic GO to the hydrophobic PET substrates, the PET surface was pre-treated with a plasma $\left(3 \% \mathrm{O}_{2} / \mathrm{Ar}\right.$ ) to yield a hydrophilic PET. The water contact angles (WCAs) exhibited a sharp decrease from $62.37^{\circ}$ to $15.14^{\circ}$ on the PET substrate and from $53.86^{\circ}$ to $20.65^{\circ}$ on the AgNW/PET substrate (Fig. 1b). The hydrophilicity on the surface of PET could be controlled with an exposed time of the plasma. As the longer plasma treatment would produce more polar oxygen groups on the surface of the film, an electrostatic interaction 

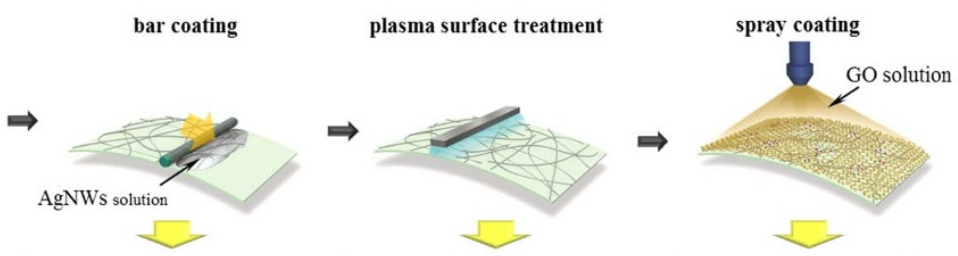

b
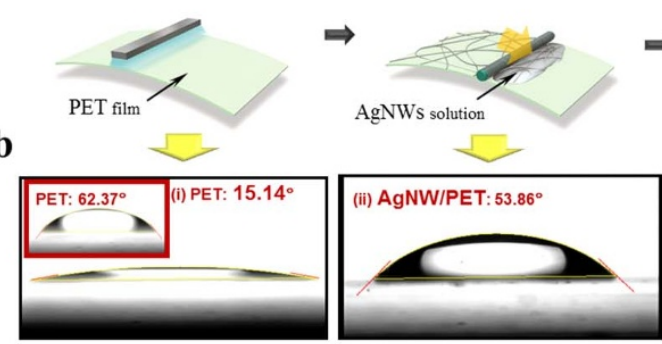

c
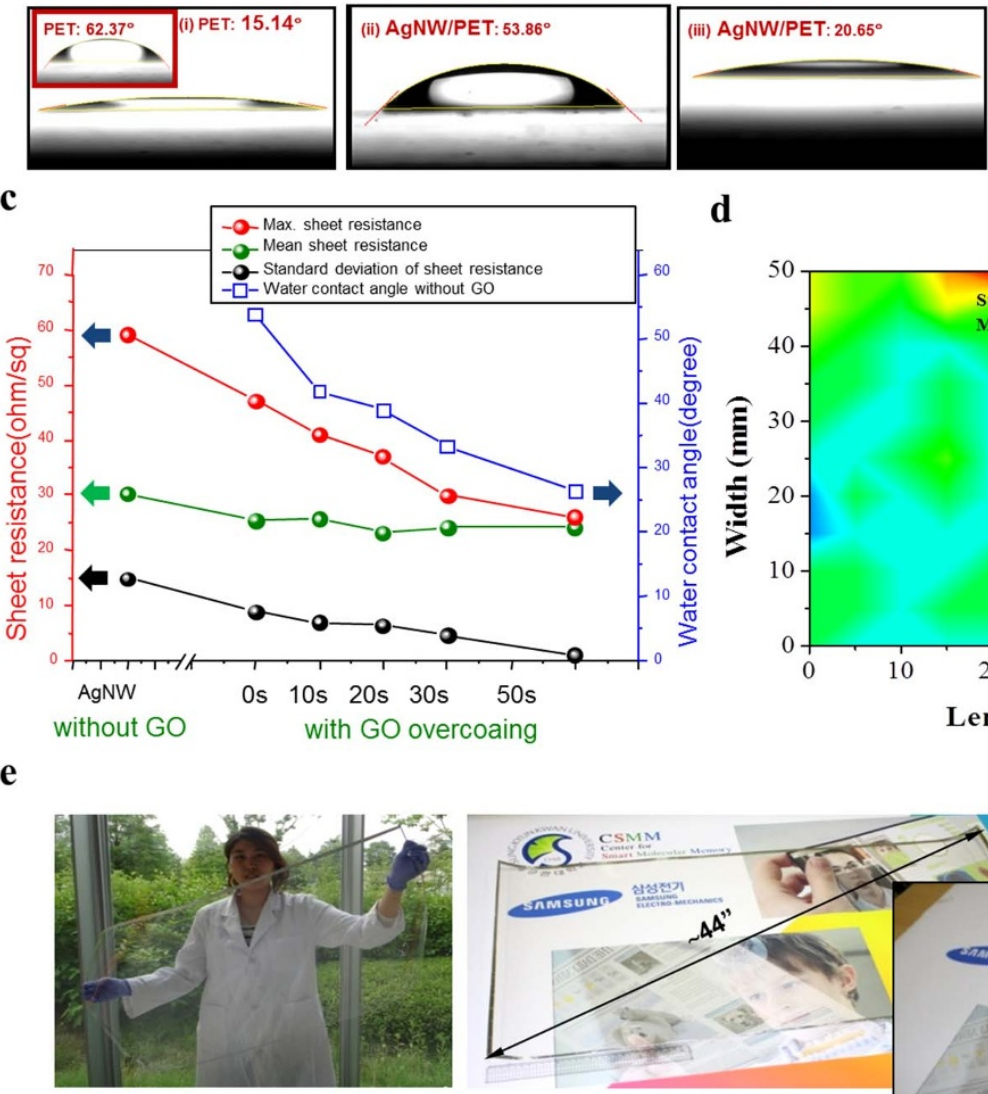

(iv) GO/AgNW/PET: $9.60^{\circ}$

d
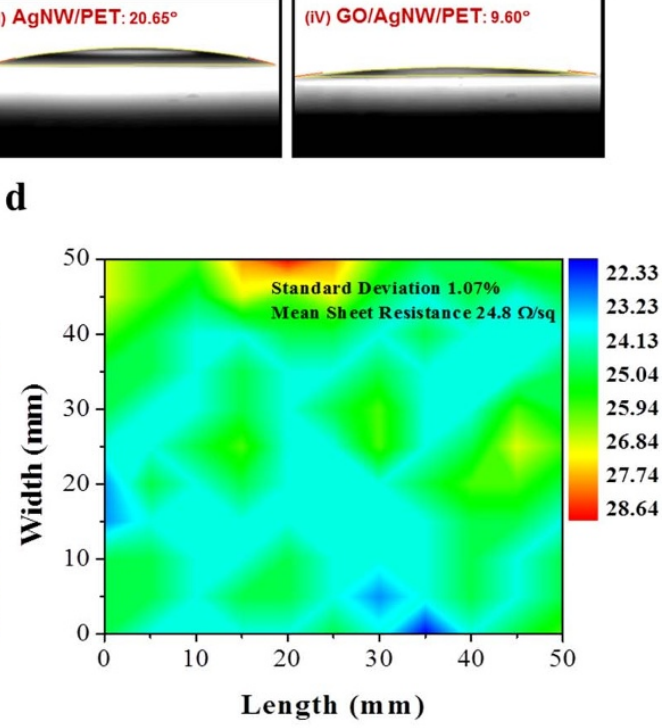

22.33
23.23

24.13

27.74

28.64

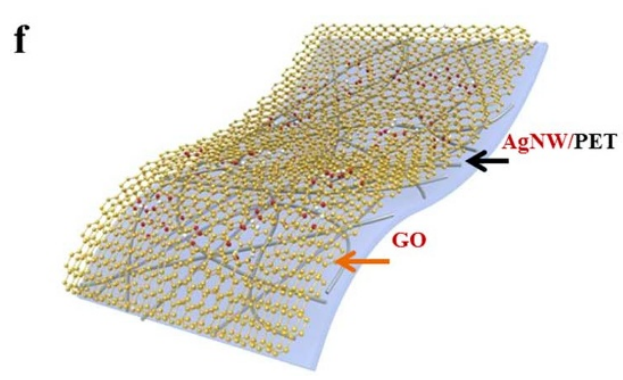

Figure $1 \mid$ (a). Schematic illustration of the key steps involved in the fabrication of the GO/AgNW/PET (or glass) film. (b). Changes in the water contact angles at the various fabrication steps: (i) $3 \% \mathrm{O}_{2} / \mathrm{Ar}$ plasma treatment; (ii) $\mathrm{AgNW}$ ink is bar or spray coated, then dried at $140^{\circ} \mathrm{C}$ for $4 \mathrm{~min}$; (iii) $3 \% \mathrm{O}{ }_{2} / \mathrm{Ar}$ plasma treatment; (iv) GO suspension is spray coated, and then dried at $140^{\circ} \mathrm{C}$ for $4 \mathrm{~min}$. (c). Changes of water contact angles, maximum sheet resistances, mean sheet resistances, and standard deviation of AgNW/PET and GO/AgNW/PET film according to exposed time of plasma treatment on the PET. (d). Spatial distribution of the GO/AgNW/PET film's sheet resistance $\left(50 \times 50 \mathrm{~mm}^{2}\right)$. (e). Flexible transparent electrode produced by spray coating with a large area $\left(100 \times 50 \mathrm{~cm}^{2}, \sim 44\right.$ ” diagonal). The inset of the right image shows a rolled GO/AgNW/PET film. (f). Schematic structure of flexible GO/AgNW/PET.

between hydrophilic PET substrate and hydrophilic GO nanosheets would be increased. As expected, the longer exposed time from 0 to 50 seconds gave a higher hydrophilicity that was confirmed with water contact angle (WCA) from $53.9^{\circ}$ to $26.3^{\circ}$, respectively, and the maximum sheet resistances covered with $\mathrm{GO}$ nanosheets decreased due to increased contact points of AgNWs by an enhanced adhesive force caused by the increased electrostatic interaction between hydrophilic PET substrate and hydrophilic GO nanosheets.
Characterization of 2D GO/AgNW/PET film. The mean sheet resistances measured at 11 different places also decreased over all. Surprisingly, with GO OCL nanosheets and longer exposed plasma treatment, the mean standard deviation (SD) of the sheet resistances of GO/AgNW/PET film sharply decreased and provided a high uniform sheet resistance over large area (Fig. 1c and Table 1). As we know, the internal haze is directly related with diameter of nanowires while the external haze is related with uniformity of the film and gaps between the $\mathrm{AgNWs}^{14}$. With high uniformity of the GO 
Table 1 | Changes of water contact angles without GO OCL, Maximum and mean sheet resistances, and their standard deviation of sheet resistance measured at 11 different places

\begin{tabular}{|c|c|c|c|c|}
\hline Plasma treatement time (sec) & Water contact angle (degree) ${ }^{a}$ & Maximum $R_{s h}{ }^{b}(\Omega / s q)$ & Mean $R_{\text {sh }}{ }^{c}(\Omega / s q)$ & $\mathrm{SD}^{d}(\%)$ \\
\hline untreated & - & 59 & 30.31 & 14.93 \\
\hline 0 & 53.86 & 47 & 25.54 & 9.04 \\
\hline 10 & 41.94 & 41 & 25.63 & 6.97 \\
\hline 30 & 33.39 & 30 & 24.27 & 4.63 \\
\hline 50 & 26.33 & 26 & 24.18 & 1.03 \\
\hline
\end{tabular}

Water contact angles for PET surface untreated and treated by $3 \% \mathrm{O}_{2} /$ Ar plasma.

${ }^{b}$ Maximum sheet resistance from Fig. 1c.

"Mean sheet resistance measured at 11 different places.

dStandard deviation of sheet resistance from Fig. 1c.

coated film and a close gap between AgNWs that can reduce a light scattering effect, total haze that is an internal plus external haze decreased with a sharp reduction of external haze.

Surprisingly, the novel GO/AgNW/PET TCF exhibited superior properties, including a low $R_{\text {sh }}$ value (average $\sim 24.8 \Omega /$ sq); high durability (over 10,000 cycles at a bending radius of $\sim 2 \mathrm{~mm}$ ); good chemical, corrosion, thermal, and adhesive stabilities; high transparency ( $T>92 \%$ at $550 \mathrm{~nm}$ ) without an anti-reflective layer; low haze (low mean reflectance, $R(\mathrm{w}) \sim 2.7 \%$ in the visible range); and a neutral colour $\left(L^{*} \sim 95.16, a^{*} \sim-0.47\right.$, and $b^{*} \sim 2.47$ ) (Fig. S4 and Table $\mathrm{S} 1)$. The novel TCF, which can be a $1^{\text {st }}$ prototype for commercial products, also exhibited optical uniformity with a $\sim 0.1 \%$ standard deviation over an A4 (or $1000 \times 500 \mathrm{~mm}^{2}$ ) area at $550 \mathrm{~nm}$ (Fig. S1). The large-area TCF film (diagonal line, $\sim 44$ inches, Figs. 1e, S2 and Supplementary Movie S1) exhibited a uniform $R_{\text {sh }}$ value $(24.8 \Omega / \mathrm{sq}$ with a $1.07 \%$ standard deviation, averaged over 121 points with a 5$\mathrm{mm}$ lattice over an area of $50 \mathrm{~mm}^{2}$ (Figs. 1d and S3)). Fig. 1f illustrated a highly flexible film structure. Our GO/AgNW/PET TCF can be used in large-area capacitive TSP, OLED and LCD displays and in solar cells that require low $R_{\text {sh }}$ values $(<30 \Omega /$ sq and $\mathrm{T}>90 \%$ ), whereas others (e.g., flexible transparent carbon nanotubes ${ }^{15}$, reduced GO thin films ${ }^{16}$ and graphene ${ }^{17}$ electrodes grown via chemical vapour deposition) with relatively high $R_{\mathrm{sh}}$ values are limited to resistive or small-sized capacitive TSPs.

\section{Discussion}

Scanning electron microscopy (SEM), atomic force microscopy (AFM) and optical microscopy (OM) (Fig. S5) were used to investigate the adhesion between the GO and the AgNW/PET (or glass). Magnified SEM (Figs. 2a and S6) and AFM images of the GO/AgNW (Figs. 2b and S7) reveal that the individual AgNW is closely packed between the GO nanosheets and the PET substrate to provide strong physical adhesion between the AgNWs. The AFM image clearly showed that the surface roughness of the GO/AgNW film decreased sharply from 14.297 to $4.848 \mathrm{~nm}$, indicating uniform and smooth surface by GO layer (Fig. S7), which results in the high transmittance ( $\mathrm{T}>92 \%$, Fig. 2c), and low optical reflectivity of the GO/AgNW/ PET (mean $2.72 \%$ ) with a broad plasmon peak at $\sim 410 \mathrm{~nm}$-the transverse plasmon band-and a shoulder peak at approximately $350 \mathrm{~nm}$ (Fig. 2c (inset) $)^{18}$. The Raman spectra indicated that the $G$ band of the GO/AgNW/glass over the GO/glass shifted from $1593 \mathrm{~cm}^{-1}$ to $1600 \mathrm{~cm}^{-1}\left(\Delta=7 \mathrm{~cm}^{-1}\right)$ (Fig. $\left.2 \mathrm{~d}\right)$. The XPS spectra revealed that the $\mathrm{Ag} 3 \mathrm{~d}_{3 / 2}$ peak arising from the $\mathrm{GO} / \mathrm{AgNW}$ over AgNW shifted from 367.2 to $368.7 \mathrm{eV}(\Delta J=1.5 \mathrm{eV})$ because of a charge transfer from the metallic AgNW to the localised $s p^{2}$ domains of the GO sheet (Figs. 2e and S8-9, Table S2) ${ }^{19}$. The $I_{2 \mathrm{D}} / I_{\mathrm{G}}$ ratio in the Raman spectra (Fig. 2d) is sensitive to the charge doping between the AgNW and the GO because of a charge transfer driven by the difference in the workfunction ${ }^{19,20}$. The $I_{2 \mathrm{D}} / I_{\mathrm{G}}$ ratio obtained with a single Lorentzian fitting increased from $\sim 0.12$ to $\sim 0.24$. The $2 \mathrm{D}$ micro-Raman maps with areas of $193 \times 35 \mu \mathrm{m}^{2}$ further confirmed the uniformity of a few GO layers (blue region) and AgNW islands (yellow to red regions) in the film (Fig. 2f), suggesting that the GO sheets adhered tightly to the hydrophilic PET surfaces owing to the electrostatic interactions between the hydroxyl groups on the basal plane of the GO sheets and the hydrophilically pre-treated PET surfaces $^{21}$. The mean $R_{\mathrm{sh}}$ values of the AgNW/PET films prepared with different $\mathrm{AgNW}$ concentrations changed with or without GO and before or after plasma treatment, as shown in Fig. 3a and Fig. $\mathrm{S} 10$, respectively. The mean $R_{\mathrm{sh}}$ values of the film with the GO OCL decreased from $\sim 27,58.8,148.8,231$, and 350 to $23.8,31,69,106.5$, and $164 \Omega \cdot \mathrm{sq}^{-1}$ compared with those without the GO OCL ${ }^{22}$. After coating the AgNW/PET with the GOs, an extremely low mean $R_{\text {sh }}$ value, $23.8 \Omega \cdot \mathrm{sq}^{-1}$, was observed, which is almost identical to or better than that of graphene prepared by chemical vapour deposition on metal grids ${ }^{23}$. Therefore, the strong adhesion between the GO OCL and the PET substrate is likely the primary factor in the improved conductivity of the AgNW. Fig. 3b presents the results of the chemical, thermal, and corrosion stability tests on AgNW/ $\mathrm{PET}$ and GO/AgNW/PET after exposure to water for $10 \mathrm{~min}$, acetone for $10 \mathrm{~min}$, isopropyl alcohol for $10 \mathrm{~min}$, or $\mathrm{H}_{2} \mathrm{~S}$ for $20 \mathrm{~min}$. Despite the extreme environment, the electrical conductivity of the GO/AgNW/PET, especially for anti-sulphuration, was excellent and was superior to that of the AgNW/PET. In addition, though the GO/ AgNW/PET was exposed to air for two months, it had excellent stability in air at high relative humidity during daylight, as shown in Figure 3c. In contrast, the AgNW/PET without GO OCL degraded significantly due to its ease of oxidation. The current-voltage (I-V) characteristics between two Au electrodes fabricated on the GO/ AgNW/PET (Fig. S12) exhibited linearity at room temperature, which suggests a good ohmic contact or a linear direct tunnel junction (Figs. 3d inset and S11). The current stability in air was also excellent under an externally applied voltage of $1.5 \mathrm{~V}$ for 20,000 sec. No degradation occurred in the electrical current and no change occurred in the colour of the film (Fig. 3d). Electro-mechanical durability is another important feature of flexible, transparent electrodes. The persistent $R_{\text {sh }}$ value after coating the AgNW/PET with GO arises from the strong adhesion resulting from the hydrophilic electrostatic interaction between the hydrophilic GO nanosheets and the hydrophilic PET surface. This interaction resulted in the AgNWs tightly adhering to the PET substrate with a strong capillary force, which provided large contact surface areas allowing the AgNWs to closely adhere to the GO OCL. The adhesion between the GO sheets and the AgNW/PET was investigated (Figs. 4a, 4b, S13 and S14). 3M Scotch tape applied with finger pressure was unable to detach the AgNWs from the GO/AgNW/PET device (Figs. 4a and S14). In contrast, without the GO layers, the majority of the AgNWs was able to be peeled-off (Figs. 4a and S13). The low $R_{\text {sh }}$ value of the AgNW film with the GO OCL was maintained even under an air atmosphere. These results suggested that the GO nanosheets have excellent mechanical adhesion to both sides of the plasma-treated plastic (or glass) substrate and to the AgNWs. A silver paste on the GO/ 


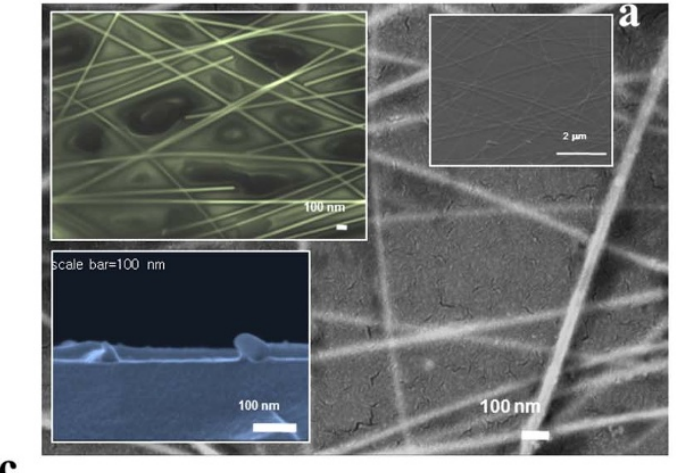

c
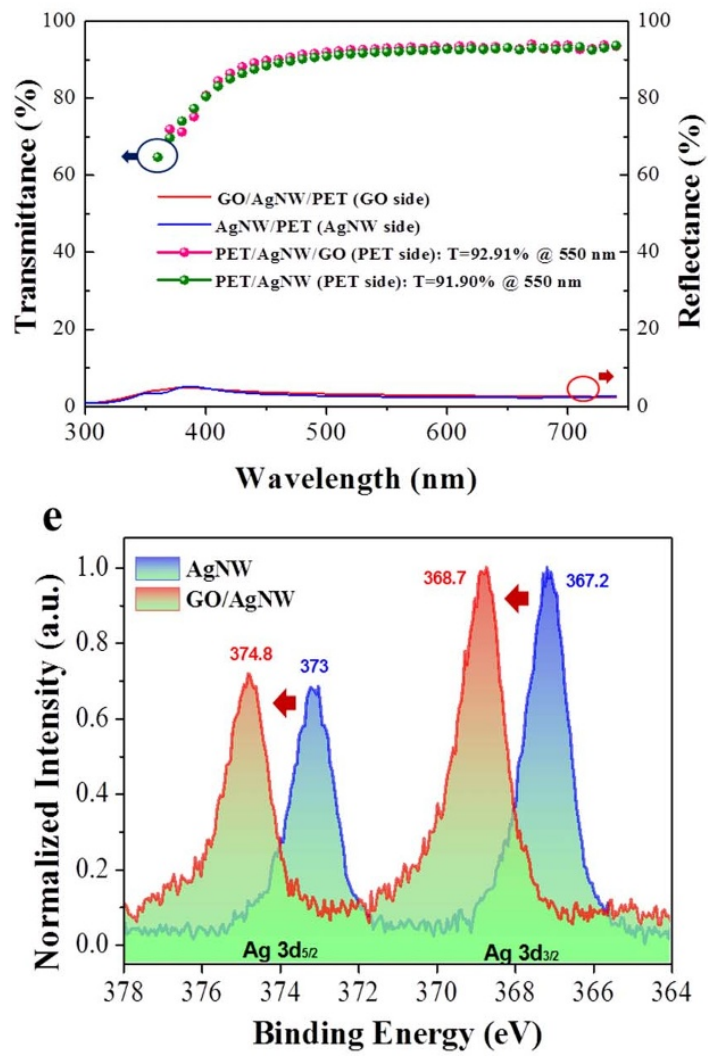

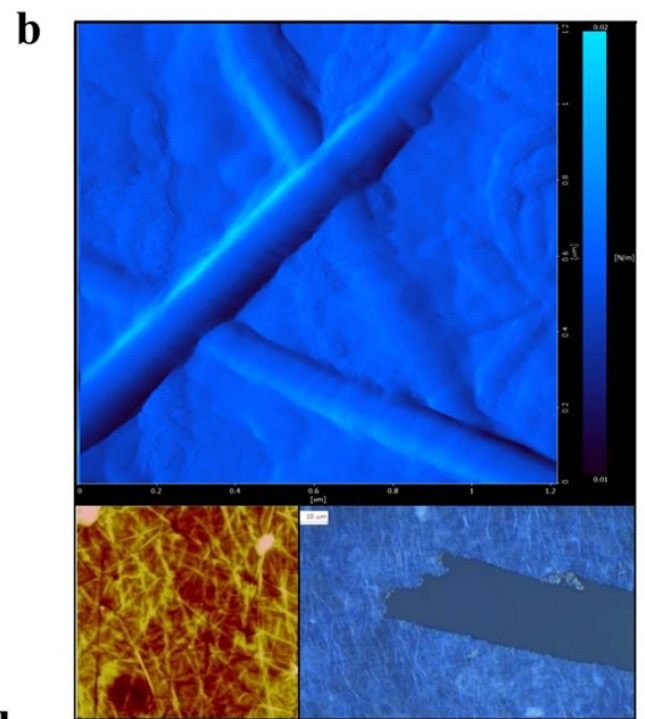

d
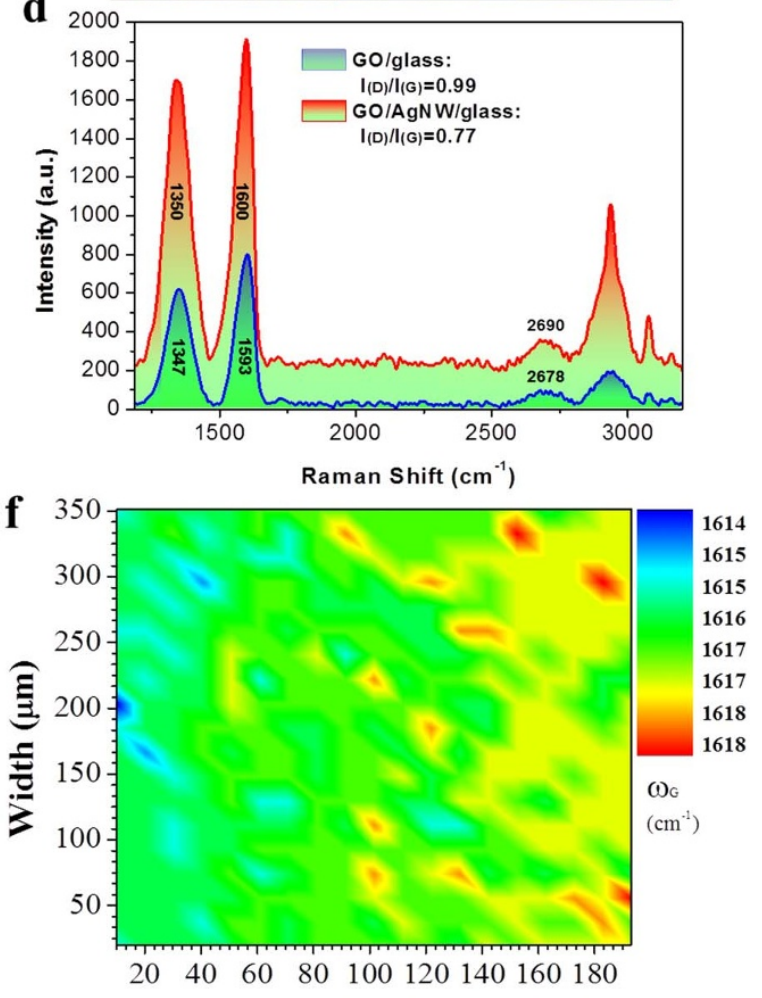

Length $(\mu \mathrm{m})$

Figure $2 \mid$ (a). SEM image of the AgNW network covered by GO nanosheets (top-left inset). A highly resolved cross-sectional SEM image of a GO/AgNW/ glass revealing the GO over-coating layers on the AgNW networks (bottom-left inset). (b). A high-magnification and a low-magnification AFM image of a $\mathrm{GO} / \mathrm{AgNW} /$ glass substrate (bottom-left, $10 \times 10 \mathrm{~mm}^{2}$ ). The surface roughness increased from 7.62 to $12.67 \mathrm{~nm}$ with a spray coating of GO. A scratched optical image (bottom-right). (c). Optical transmittance ( $\mathrm{T}>92 \%$ at $550 \mathrm{~nm}$ ) and reflection spectra of AgNW/PET (blue line) and GO/AgNW/PET (red line), respectively. (d). Raman spectra before and after coating the AgNW/glass film with GO. (e). XPS spectra of the Ag $3 \mathrm{~d}$ band of each film. (f). Spatial Raman map of the G line taken for the GO on the AgNW/PET film.

AgNW/PET film for the silver bus bar on each side of the active area of the capacitive TSP did not detach. The $R_{\text {sh }}$ value of the GO/AgNW/ PET was examined as a function of the bending angle to determine the foldability of the flexible electrodes. At most, the $R_{\mathrm{sh}}$ increased only $\sim 1.4 \%$ for the $0-148^{\circ}$ bending angles, indicating excellent durability (Fig. 4c). The fully folded semi-circular GO/AgNW/PET exhibited a persistent current flow (Supplementary Movie S2). The bendability of the as-prepared GO/AgNW/PET was systematically examined using an in situ bending test in which two copper electrodes simultaneously rotate (Fig. 4d and Supplementary Movie S3).
The highly conductive GO/AgNW/PET exhibited a harsh stress and strain in the $\sim 20^{\circ}$ gripping region with a $\sim 2 \mathrm{~mm}$ curvature radius $(r)$ under a bending rate of $1 \mathrm{~Hz}$. With over 10,000 bending cycles ( $\sim 2 \mathrm{~mm}$ bending radius) between the compression (inner bending) and tension (outer bending) modes, the newly developed GO/AgNW/PET film exhibited excellent bendability with an almost constant sheet resistance, even with a 2 -fold conductance loss. The resistance change under the bending modes can be explained by a complex change in the geometry between the slipping and delamination of the AgNW junctions (Fig. S15) ${ }^{24}$. 

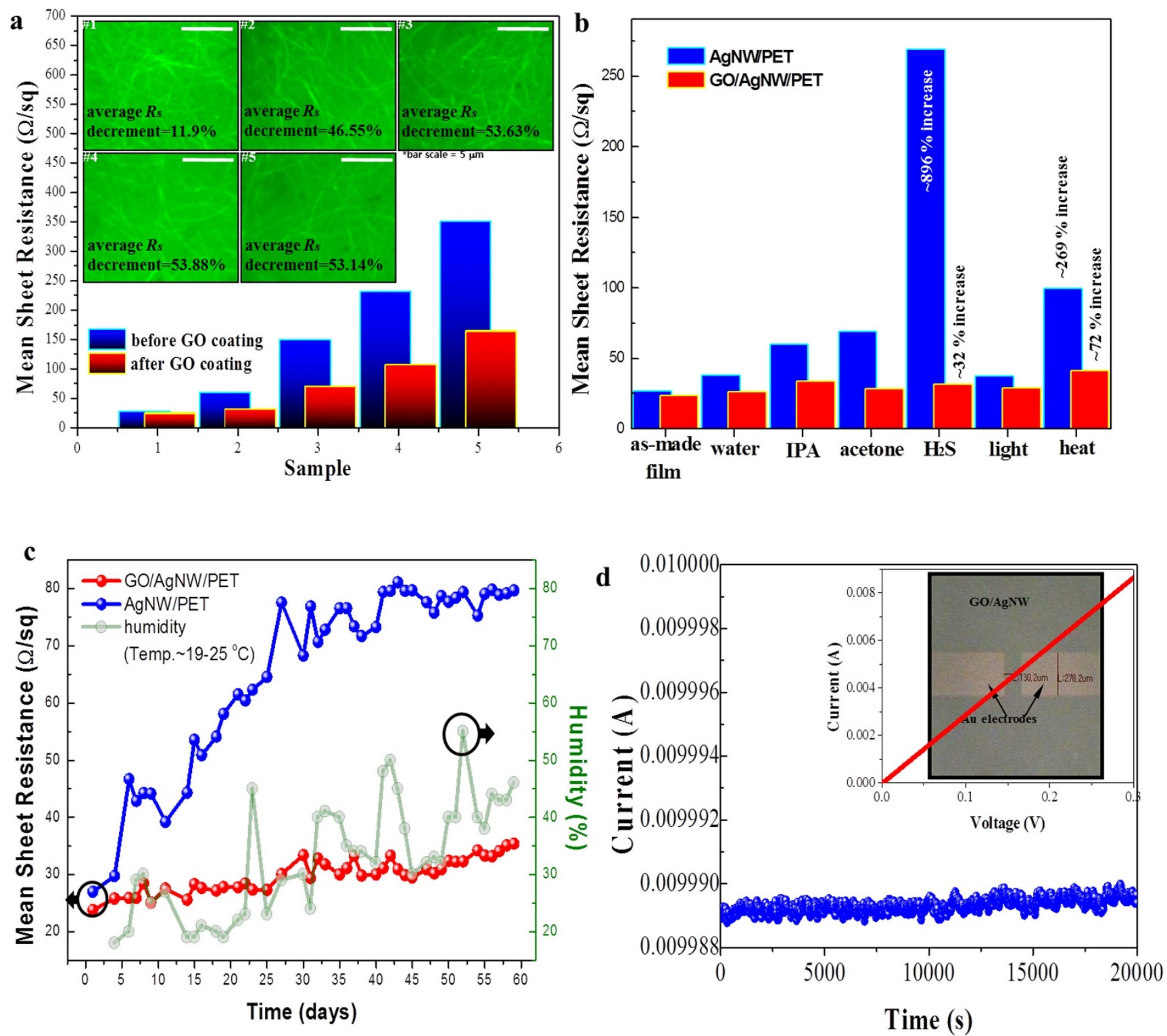

Figure 3 The chemical and electrical stabilities of the GO/AgNW/PET film. (a). Changes in the average $\mathrm{R}_{\mathrm{sh}}$ values of the different densities of AgNW/ PET films after coating with GO. The inset contains OM images of each sample. (b). Chemical, thermal, and corrosion stability of the AgNW/PET and $\mathrm{GO} / \mathrm{AgNW} / \mathrm{PET}$ films after exposure to various solvents and gases. (c). Plot of the mean $\mathrm{R}_{\mathrm{sh}}$ versus time for the AgNW/PET and GO/AgNW/PET films exposed to air for two months. (d). Plot of the current changes over time for GO/AgNW/PET with an applied voltage of $1.5 \mathrm{~V}$. The inset is a schematic device structure of $\mathrm{Au} / \mathrm{GO} / \mathrm{AgNW} / \mathrm{PET}$; the currents from two Au electrodes as a function of applied voltage are linear, which indicates excellent ohmic transport through the GO over-coating layer.

We have demonstrated that 2D insulating and hydrophilic GO nanosheet can be used as a novel adhesive OCL on the hydrophilic AgNW/PET. With GO OCL nanosheets and longer exposed plasma treatment, the maximum and mean sheet resistances decreased and the uniformity of the film increased, resulting in high quality AgNW film with a low haze. In conclusion, with ultra-thin GO nanosheet as the OCL, all of the important hurdles for the real commercialization, including high haze, low transparency, high $R_{\mathrm{sh}}$ values and mechanical instability, were simultaneously overcome, thereby producing the large area, flexible, highly transparent conductive GO/AgNW/ PET film. All of the components in this system were produced using inexpensive solution processes. These wet-processable, extremely stable, mechanically compliant and physically robust TCF can be applied to large-area, flexible displays and solar cells, optical/electronic biosensors, energy nanogenerators, and transparent energy storages as a charge collectors.

\section{Methods}

Materials. All of the AgNW/PET films were carefully prepared by Samsung ElectroMechanics (Suwon, Korea). The GO nanosheets described in our previous publication $^{1}$ (flake size $\approx 0.05 \mathrm{~mm}$, single layer, concentration $>\sim 80 \%$ ) were prepared using a modified Hummers method ${ }^{2}$. $\mathrm{H}_{2} \mathrm{~S}$ was purchased from Aldrich for the corrosion test.

Preparation of GO/AgNW/PET film. For a control experiment, PET substrate was not pre-treated to observe the role of $\mathrm{GO}$ on the surface of silver nanowire. PET substrate was pre-treated with $3 \% \mathrm{O}_{2} /$ Argon plasma for 10, 20, 30, 40 and 50 seconds to give $41.9^{\circ}, 38.9^{\circ}, 33.3^{\circ}$ and $26.3^{\circ} \mathrm{WCAs}$, respectively. For a bar coating technique, AgNW ink was pipetted in a line at the top of the hydrophilic PET substrate (PET pieces $=10 \times 10 \mathrm{~cm}^{2}$ or A4 size). Then, a Meyer rod (\#10) was a quickly (within 1 second) pulled down over the AgNW ink by hand. Different densities of AgNW inks on the hydrophilic PET substrate were obtained with a dilution by using isopropyl alcohol. The obtained-AgNW/PET film was then dried in a $140^{\circ} \mathrm{C}$ oven for $4 \mathrm{~min}$. AgNW/PET film was treated once more with plasma $\left(3 \% \mathrm{O}_{2} /\right.$ argon) for 60 seconds to give $20.65^{\circ} \mathrm{WCA}$ and GO nanosheets in aqueous solution (concentration $=27.5 \mathrm{mg} / \mathrm{L}$ ) were a 3 -times sprayed on the hydrophilic AgNW/PET 

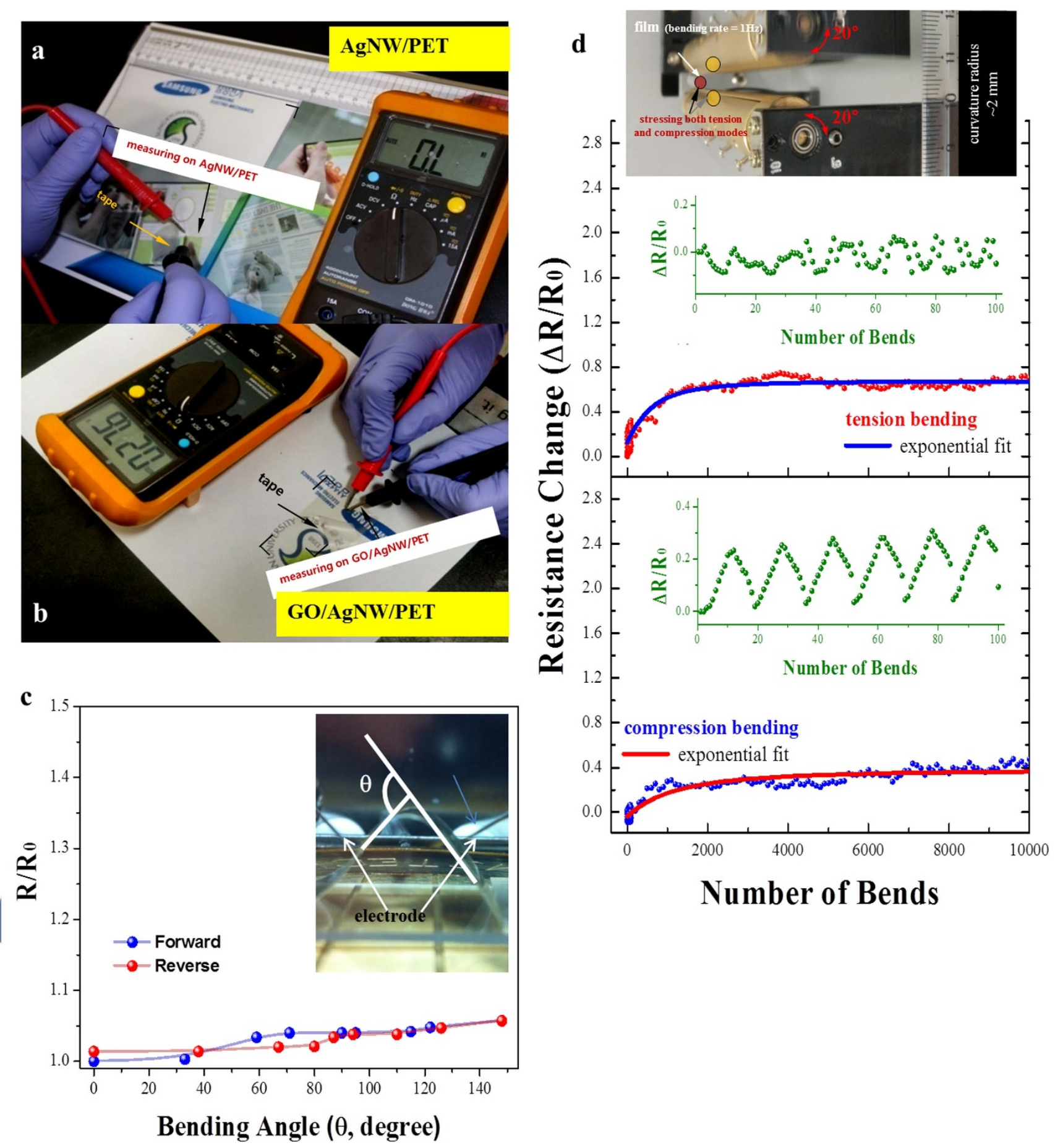

Figure 4 | Electro-mechanical stability of the GO/AgNW/PET film. (a). Mechanical adhesion test for AgNW/PET and (b) GO/AgNW/PET with 3M Scotch ${ }^{\circledR}$ tape. The sheet resistance was not observed due to the detachment of the AgNWs from the AgNW/PET film (a), but was observed without detachment of the AgNWs from the GO/AgNW/PET film. (c). Bending test of the GO/AgNW/PET film, which shows a minimal change in R/R $\mathrm{R}_{0}$ with an increased bending angle. The right inset provides an image of a folded film that is set up for the bending test. (d). The electrical stability test measured before and after the in situ bending test to obtain the two probes' sheet resistances in the GO/AgNW/PET film. To release the high stress and strain at the gripping region of the films, an initial position was set with an orientation angle of $\sim 20^{\circ}$ (upper image in $\mathrm{d}$ ).

film to give $9.6^{\circ}$, and then dried in a $140^{\circ} \mathrm{C}$ oven for $4 \mathrm{~min}$. The resulting sheet resistances of each film were measured at 11 different points.

Exposure tests for anti-sulphuration of GO/AgNW/PET film. Exposure tests in bell-jar of diameter $10 \mathrm{~cm}$ and length $25 \mathrm{~cm}$ were performed to evaluate the ability of anti-sulphuration using two different kinds of films. Both AgNW/PET film and GO/ AgNW/PET film were incubated in glass dessiccator with inner diameter $\Phi 180 \mathrm{~mm}$. 99.5\% hydrogen sulfide gas (Aldrich Co.) was then allowed to flow into the reaction chamber with the assistance of an argon carrier gas, which is provided at a flow rate of 10 SCCM. Finally, the films were exposed to 50 SCCM of the diluted $\mathrm{H}_{2} \mathrm{~S}$ for $20 \mathrm{~min}$.
The sheet resistance was checked after rinsing with de-ionized water and blowing to be dried with nitrogen gas.

Fabrication of $\mathbf{A u} / \mathrm{GO} / \mathrm{AgNW} / \mathrm{PET}$ film. To measure the resistance and electrical stability of $\mathrm{Au} / \mathrm{GO} / \mathrm{AgNW} / \mathrm{PET}$ film, each $\mathrm{Au} / \mathrm{GO} / \mathrm{AgNW} / \mathrm{PET}$ sample was prepared by the thermal evaporation of gold top electrodes with a distance of $\sim 130 \mu \mathrm{m}$ between the gold electrodes separated by a shadow mask. The sheet resistances of these films were measured with a Keithley 4200 semiconductor characterisation system at room temperature under atmosphere. 
Bending test of GO/AgNW/PET film. The bendabilities of GO/AgNW/PET film were analysed using a bending test system (ZBT-200, Z-Tech, Korea). Two terminal measurements were used to monitor the change in resistance of each AgNW/PET and $\mathrm{GO} / \mathrm{AgNW} / \mathrm{PET}$ film with various bending angle from 0 to 150 degree. The curvature radius of the bended film was about $2 \mathrm{~mm}$. When the film was straight, the sheet resistance were recorded.

Characterisation of samples. The morphologies of each sample were characterised by SEM (FEI NOVA ${ }^{\mathrm{TM}}$ NanoSEM 200 or JEOL JSM-7600F), AFM (Digital Instruments D3100, PSIA XE-100 or Agilent 5500 AFM/SPM), OM (Olympus BX51, Microscopes Inc. wm003900a S39a, or Sometech Vision Camscope), and X-ray diffraction (Ultima IV, Rigaku). WCA measurements were performed to determine the wettability of the film surfaces using an SEO Phoenix 300 microscope. The optical transmission and reflectance profiles of the films were recorded on a Varian Cary 5000 UV-Vis spectrophotometer and a Konica Minolta Horizontal Spectrophotometer CM-3600D. The haze value of the film (defined as the ratio between the diffuse and total transmission, $\Delta \mathrm{T} / \mathrm{T}$ ) was measured using an NDH 5000 haze meter (Nippon Denshoku Industries Co., Ltd.). The colorimetry of the films was evaluated using a Konica Minolta Horizontal Spectrophotometer CM-3600D. Linear and 2D Raman spectra were measured on a Renishaw RM 1000-In Via Raman microscope equipped with a 513-nm laser and a Jobin Yvon/Horiba LabRAM Aramis Raman microscope equipped with a 513-nm laser (mapping software: LabSpec5), respectively. A VG Microtech ESCA 2000 equipped with a Mg/Al source was used to analyse the surface chemical bonding states. The $R_{\mathrm{sh}}$ value of each sample was measured using a four-point probe surface resistivity meter (R-CHEK 4-point sheet resistance meter, model RC2175). The $R_{\mathrm{sh}}$ values were obtained by averaging the measurements of at least 10 points for each sample.

1. Moon, I. K. et al. Reduced Graphene Oxide by Chemical Graphitization. Nature Communications 1, 73 (2010).

2. Hummers, W. S. \& Offeman, R. E. Preparation of graphitic oxide. J. Am. Chem. Soc. 80, 1339 (1958).

3. Gómez-Navarro, C. et al. Atomic structrue of reduced graphene oxide. Nano Lett. 10, 1144-1148 (2010).

4. Lightcap, I. V., Kosel, T. H. \& Kamat, P. V. Achoring semiconductor and metal nanoparticles on a two-dimensional catalyst mat. storing and shuttling electrons with reduced graphene oxide. Nano Lett. 10, 577-583 (2010).

5. Kholmanov, I. et al. Nanostructured Hybrid Transparent Conductive Films with Antibacterial Properties. ACS Nano, 6, 5157-5163 (2012).

6. Hecht, D. S., Hu, L. \& Irvin, G. Emerging transparent electrodes based on thin films of carbon, graphene, and metallic nanostructures. Adv. Mater. 23, 1482-2699 (2011)

7. Ju, S. et al. Fabrication of fully transparent nanowire transistors for transparent and flexible electronics. Nat. Nanotechnol. 2, 378-384 (2007).

8. Huang, Y. Y. \& Terentjev, E. M. Transparent electrode with a nanostructured coating. ACS Nano. 5, 2081-2089 (2011)

9. Garnett, E. C. et al. Self-limited plasmonic welding of silver nanowire junctions. Nat. Mater. 11, 241-249 (2012).

10. Tokuno, T. et al. Fabrication of silver nanowire transparent electrodes at room temperature. Nano Res. 4, 1215-1222 (2011).

11. Zhu. R. et al. Fused silver nanowires with metal oxide nanoparticles and organic polymers for highly transparent conductors. ACS Nano 5, 9877-9882 (2012).

12. Gelves, G. A., Lin, B., Sundaraj, U. \& Haber, J. A. Low electrical percolation threshold of silver and copper nanowires in polystyrene. Adv. Funct. Mater. 16, 2423-2430 (2006).
13. Hu, L., Kim, H. S., Lee, J.-Y., Peumans, P. \& Cui, Y. Scalable coating and properties of transparent, flexible, silver nanowire electrodes. ACS Nano 4, 2955-2963 (2010).

14. Szanyi, Janos. The origin of haze in CVD tin oxide thin films.. Applied Surface Science 185, 161-171 (2002).

15. Hecht, D. S. et al. Carbon-nanotube film on plastic as transparent electrode for resistive touch screens. Journal of the SID 17, 941-946 (2009).

16. Wang, J. et al. Rod-coating: towards large-area fabrication of uniform reduced graphene oxide films for flexible touch screens. Adv. Mater 24, 2874-2878 (2012).

17. Bae, S. et al. Roll-to-roll production of 30-inch graphene films for transparent electrodes. Nat. Nanotechnol. 5, 574-578 (2010).

18. Y. Sun, Y., Gates, B., Mayers, B. \& Xia, Y. Crystalline silver nanowires by soft solution processing. Nano Lett. 2, 165-168 (2002).

19. Deng, Z., Chen, M. \& Wu, L. Novel method to fabricate $\mathrm{SiO}_{2} / \mathrm{Ag}$ composite spheres and their catalytic, surface-enhanced Raman scattering properties. J. Phys. Chem. C. 111, 11692-11698 (2007).

20. Pisanan, S. et al. Breakdown of the adiabatic Born-Openheimer approximation in graphene. Nat. Mater. 6, 198-201 (2007).

21. Szabó, T. Evolution of surface functional gropus in a series of progressively oxidized graphite oxides. Chem Mater. 18, 2740-2749 (2006).

22. Gómez-Navarro, C. et al. Electronic transport properties of individual chemically reduced graphene oxide sheets. Nano Lett 7, 3499-3503 (2011)

23. Zhu, Y., Sun, Z., Yan, Z., Jin, Z. \& Tour, J. M. Rational design of hybrid graphene films for high-performance transparent electrodes. ACS Nano 5, 6472-6479 (2011).

24. Park, S.-I. et al. Theoretical and experimental studies of bending of inorganic electronic materials on plastic substrates. Adv. Funct. Mater 18, 2673-2684 (2008).

\section{Acknowledgements}

This work was supported by the Creative Research Initiatives (project title: Smart Molecular Memory) of MEST/NRF. The authors are grateful to Mrs. E.H. Hwang for AFM observations.

\section{Author contributions}

I.K.M. W.C.K. and H.L. designed the research and wrote the manuscript. I.K.M., J.I.K. and HL.L. performed the experiments and analysed the data. K.H. discussed the results.

\section{Additional information}

Supplementary information accompanies this paper at http://www.nature.com/ scientificreports

Competing financial interests: The authors declare no competing financial interests.

License: This work is licensed under a Creative Commons

Attribution-NonCommercial-NoDerivs 3.0 Unported License. To view a copy of this license, visit http://creativecommons.org/licenses/by-nc-nd/3.0/

How to cite this article: Moon, I.K. et al. 2D Graphene Oxide Nanosheets as an Adhesive Over-Coating Layer for Flexible Transparent Conductive Electrodes. Sci. Rep. 3, 1112, DOI:10.1038/srep01112 (2013) 